\title{
Integrable Nonlocal Reductions
}

\author{
Metin Gürses * \\ Department of Mathematics, Faculty of Science \\ Bilkent University, 06800 Ankara - Turkey \\ Asli Pekcan ${ }^{\dagger}$ \\ Department of Mathematics, Faculty of Science \\ Hacettepe University, 06800 Ankara - Turkey
}

\begin{abstract}
We present some nonlocal integrable systems by using the Ablowitz-Musslimani nonlocal reductions. We first present all possible nonlocal reductions of nonlinear Schrödinger (NLS) and modified Korteweg-de Vries (mKdV) systems. We give soliton solutions of these nonlocal equations by using the Hirota method. We extend the nonlocal NLS equation to nonlocal Fordy-Kulish equations by utilizing the nonlocal reduction to the Fordy-Kulish system on symmetric spaces. We also consider the super AKNS system and then show that AblowitzMusslimani nonlocal reduction can be extended to super integrable equations. We obtain new nonlocal equations namely nonlocal super NLS and nonlocal super mKdV equations.
\end{abstract}

\section{Introduction}

After the publications of the Ablowitz-Musslimani works [1]-[3] on nonlocal nonlinear Schrödinger (NLS) equation there is a huge interest in obtaining nonlocal reductions of systems of integrable equations [4]-[22]. In all these works the soliton solutions and their properties were investigated by using inverse scattering techniques, by Darboux transformations, and by the Hirota direct method.

Recently we extended the nonlocal NLS equations to nonlocal Fordy-Kulish equations by utilizing the nonlocal reduction to the Fordy-Kulish system on symmetric spaces [23]. In a previous work [24] we studied the coupled NLS system obtained from AKNS scheme. By using the Hirota bilinear method we first found soliton solutions of the coupled NLS system of equations then using the Ablowitz-Musslimani type reduction formulas we obtained the soliton solutions of the standard and time T-, space S-, and space-time ST- reversal symmetric nonlocal NLS equations. Similarly, in a recent work [26] we studied the nonlocal modified Korteweg-de Vries (mKdV) equations

\footnotetext{
*gurses@fen.bilkent.edu.tr

†Email:aslipekcan@hacettepe.edu.tr
} 
which are also obtained from AKNS scheme by Ablowitz-Musslimani type nonlocal reductions. For this purpose we start using the soliton solutions of the coupled $\mathrm{mKdV}$ system found by Hirota and Iwao [27]. Then by using these solutions and Ablowitz-Musslimani type reduction formulas we obtained solutions of standard and nonlocal $\mathrm{mKdV}$ and complex $\mathrm{mKdV}(\mathrm{cmKdV})$ equations including one-, two-, and three-soliton waves, complexitons, breather-type, and kink-type waves. We used two different types of approaches in finding the soliton solutions. We gave one-soliton solutions of both types and presented only first type of two- and three-soliton solutions (see [26]).

When the Lax pair, in $(1+1)$-dimensions, is given in a Lie algebra the resulting evolution equations are given as a coupled system

$$
\begin{aligned}
q_{t}^{i} & =F^{i}\left(q^{k}, r^{k}, q_{x}^{k}, r_{x}^{k}, q_{x x}^{k}, r_{x x}^{k}, \cdots\right), \\
r_{t}^{i} & =G^{i}\left(q^{k}, r^{k}, q_{x}^{k}, r_{x}^{k}, q_{x x}^{k}, r_{x x}^{k}, \cdots\right),
\end{aligned}
$$

for all $i=1,2, \cdots, N$ where $F^{i}$ and $G^{i}(i=1,2, \cdots, N)$ are functions of the dynamical variables $q^{i}(t, x), r^{i}(t, x)$, and their partial derivatives with respect to $x$. Since we start with a Lax pair then the system (1)-(2) is an integrable system of nonlinear partial differential equations.

In the space of dynamical variables $\left(q^{i}, r^{i}\right)$ there exist subspaces

$$
r^{i}(t, x)=k q^{i}(t, x)
$$

or

$$
r^{i}(t, x)=k \bar{q}^{i}(t, x),
$$

where $k$ is a constant and a bar over a letter denotes complex conjugation, such that the systems of equations (1)-2) reduce to one system for $q^{i}$, s

$$
q_{t}^{i}=\tilde{F}^{i}\left(q^{k}, q_{x}^{k}, q_{x x}^{k}, \cdots\right)
$$

provided that the second system (2) consistently reduces to the above system (5) of equations. Here $\tilde{F}=\left.F\right|_{r=k \bar{q}}$. Recently a new reduction is introduced by Ablowitz and Musslimani [1]-[3]

$$
r^{i}(t, x)=k q^{i}\left(\mu_{1} t, \mu_{2} x\right)
$$

or

$$
r^{i}(t, x)=k \bar{q}^{i}\left(\mu_{1} t, \mu_{2} x\right),
$$

for $i=1,2, \cdots, N$. Here $k$ is a constant and $\mu_{1}^{2}=\mu_{2}^{2}=1$. When $\left(\mu_{1}, \mu_{2}\right)=\{(-1,1)$, $(1,-1),(-1,-1)\}$ the above constraints reduce the system 11 to nonlocal differential equations provided that the second system (2) consistently reduces to the first one. If the reduction is done in a consistent way the reduced system of equations is also integrable. This means that the reduced system admits a recursion operator and bi-hamiltonian structure and the reduced system has 
$N$-soliton solutions. The inverse scattering method (ISM) can also be applied. Ablowitz and Musslimani have first found the nonlocal NLS equation from the coupled AKNS equations and solved it by ISM [2].

In our studies of nonlocal NLS and nonlocal $\mathrm{mKdV}$ equations we introduced a general method to obtain soliton solutions of nonlocal integrable equation. This method consists of three main steps:,

(i) Find a consistent reduction formula which reduces the integrable system of equations to integrable nonlocal equations.

(ii) Find soliton solutions of the system of equations by use of the Hirota direct method or by inverse scattering transform technique, or by use of Darboux Transformation.

(iii) Use the reduction formulas on the soliton solutions of the system of equations to obtain the soliton solutions of the reduced nonlocal equations. By this way one obtains many different relations among the soliton parameters of the system of equations.

In the following sections we mainly follow the above method in obtaining the soliton solutions of the nonlocal NLS and nonlocal mKdV equations.

\section{AKNS System}

When we begin with the Lax pair in $\operatorname{sl}(2, R)$ algebra and assume them as a polynomial of the spectral parameter of degree less or equal to three then we obtain the following system of evolution equations [25]:

$$
\begin{aligned}
& q_{t}=a_{2}\left(-\frac{1}{2} q_{x x}+q^{2} r\right)+i a_{3}\left(-\frac{1}{4} q_{x x x}+\frac{3}{2} q r q_{x}\right), \\
& r_{t}=a_{2}\left(\frac{1}{2} r_{x x}-q r^{2}\right)+i a_{3}\left(-\frac{1}{4} r_{x x x}+\frac{3}{2} q r r_{x}\right) .
\end{aligned}
$$

Here $a_{2}$ and $a_{3}$ are arbitrary constants.

Letting $a_{2}=1 / a$ and $a_{3}=0$ we get the coupled NLS system,

$$
\begin{aligned}
& a q_{t}=-\frac{1}{2} q_{x x}+q^{2} r \\
& a r_{t}=\frac{1}{2} r_{x x}-q r^{2}
\end{aligned}
$$

where $a$ is any constant. The corresponding recursion operator is

$$
\mathscr{R}=\left(\begin{array}{lc}
q D_{x}^{-1} r-\frac{a}{2} D_{x} & q D_{x}^{-1} q \\
-r D_{x}^{-1} r & -r D_{x}^{-1} q+\frac{a}{2} D_{x}
\end{array}\right) .
$$


One-soliton solution of the system (10)-(11) can be obtained by the Hirota method as

$$
q(t, x)=\frac{e^{\theta_{1}}}{1+A e^{\theta_{1}+\theta_{2}}}, \quad r(t, x)=\frac{e^{\theta_{2}}}{1+A e^{\theta_{1}+\theta_{2}}},
$$

where $\theta_{i}=k_{i} x+\omega_{i} t+\delta_{i}, i=1,2$ with $\omega_{1}=k_{1}^{2} / 2 a, \omega_{2}=-k_{2}^{2} / 2 a$, and $A=-1 /\left(k_{1}+k_{2}\right)^{2}$. Here $k_{1}, k_{2}, \delta_{1}$, and $\delta_{2}$ are arbitrary complex numbers.

\section{Standard and Nonlocal NLS Equations}

Standard reduction of NLS equation is $r(t, x)=k \bar{q}(t, x)$ where $k$ is a real constant. The second equation (11) is consistent if $\bar{a}=-a$. Then the NLS system reduces to

$$
a q_{t}=-\frac{1}{2} q_{x x}+k q^{2} \bar{q}
$$

Recursion operator of the NLS equation is

$$
\mathscr{R}=\left(\begin{array}{cc}
q D_{x}^{-1} \bar{q}-\frac{a}{2} D_{x} & q D_{x}^{-1} q \\
-\bar{q} D_{x}^{-1} \bar{q} & -\bar{q} D_{x}^{-1} q+\frac{a}{2} D_{x}
\end{array}\right) .
$$

There are two types of approaches to find solutions of the standard and nonlocal NLS equations. In Type 1, one-soliton solution is obtained by letting $k_{2}=\bar{k}_{1}$ and $e^{\delta_{2}}=k e^{\bar{\delta}_{1}}$ in 13 as

$$
q(t, x)=\frac{e^{\theta_{1}}}{1+A k e^{\theta_{1}+\bar{\theta}_{1}}} .
$$

In Type 2 we obtain a different solution under the constraints,

$$
\text { 1) } \left.\left.\left.\bar{a}=-a, \text { 2) } k_{1}=-\bar{k}_{1}, 3\right) k_{2}=-\bar{k}_{2}, 4\right) A k e^{\delta_{1}+\bar{\delta}_{1}}=1,5\right) A e^{\delta_{2}+\bar{\delta}_{2}}=k
$$

If we take $a=i \alpha, k_{1}=i \beta, k_{2}=i \gamma, e^{\delta_{1}}=a_{1}+i b_{1}$, and $e^{\delta_{2}}=a_{2}+i b_{2}$ for $\alpha, \beta, \gamma, a_{j}, b_{j}$ $\in \mathbb{R}, j=1,2$ one-soliton solution of standard NLS equation becomes

$$
q(t, x)=\frac{e^{i \beta x+\frac{i \beta^{2}}{2 \alpha} t}\left(a_{1}+i b_{1}\right)}{1+\frac{1}{(\beta+\gamma)^{2}} e^{i(\beta+\gamma) x+i \frac{\left(\beta^{2}-\gamma^{2}\right)}{2 \alpha} t}\left(a_{1}+i b_{1}\right)\left(a_{2}+i b_{2}\right)}, \quad \beta \neq-\gamma,
$$

and therefore

$$
|q(t, x)|^{2}=\frac{a_{1}^{2}+b_{1}^{2}}{4} \sec ^{2}\left(\frac{\theta}{2}\right)
$$

where

$$
\theta=(\beta+\gamma) x+\frac{1}{2 \alpha}\left(\beta^{2}-\gamma^{2}\right) t+\omega_{0}
$$

for $\omega_{0}=\arccos \left(\left(a_{1} a_{2}-b_{1} b_{2}\right) /(\beta+\gamma)^{2}\right)$ with $a_{1}^{2}+b_{1}^{2}=(\beta+\gamma)^{2} / k$ and $a_{2}^{2}+b_{2}^{2}=k(\beta+\gamma)^{2}$. This solution is singular for any choice of the parameters. 
Let now $r(t, x)=k \bar{q}\left(\mu_{1} t, \mu_{2} x\right)$ where $\mu_{1}^{2}=\mu_{2}^{2}=1$ and $k$ is a real constant. This is an integrable reduction, meaning that the new equation we obtain

$$
a q_{t}(t, x)=-\frac{1}{2} q_{x x}(t, x)+k q^{2}(t, x) \bar{q}\left(\mu_{1} t, \mu_{2} x\right),
$$

is integrable and the second equation $(11)$ is consistent with the first one (10) provided that $\bar{a}=$ $-\mu_{1} a$. The recursion operator of this equation is

$$
\mathscr{R}=\left(\begin{array}{cc}
k q(t, x) D_{x}^{-1} \bar{q}\left(\mu_{1} t, \mu_{2} x\right)-\frac{a}{2} D_{x} & q(t, x) D_{x}^{-1} q(t, x) \\
-k^{2} \bar{q}\left(\mu_{1} t, \mu_{2} x\right) D_{x}^{-1} \bar{q}\left(\mu_{1} t, \mu_{2} x\right) & -k \bar{q}\left(\mu_{1} t, \mu_{2} x\right) D_{x}^{-1} q(t, x)+\frac{a}{2} D_{x}
\end{array}\right),
$$

and one-soliton solution is obtained by letting $k_{2}=\mu_{2} \bar{k}_{1}$ and $e^{\delta_{2}}=k e^{\bar{\delta}_{1}}$ in 13 as

$$
q(t, x)=\frac{e^{\theta_{1}(t, x)}}{1+A k e^{\theta_{1}(t, x)+\bar{\theta}_{1}\left(\mu_{1} t, \mu_{2} x\right)}},
$$

in Type 1 approach.

In Type 2, under the constraints

$$
\text { 1) } \left.\left.\left.\left.\bar{a}=-\mu_{1} a, 2\right) k_{1}=-\bar{k}_{1} \mu_{2}, 3\right) k_{2}=-\bar{k}_{2} \mu_{2}, 4\right) A k e^{\delta_{1}+\bar{\delta}_{1}}=1,5\right) A e^{\delta_{2}+\bar{\delta}_{2}}=k
$$

we obtain a different one-soliton solution.

Nonlocal reductions of NLS system correspond to $\left(\mu_{1}, \mu_{2}\right)=\{(-1,1),(1,-1)$, $(-1,-1)\}$. Hence we have three different reductions of the NLS system (10)-(11).

1) T-Symmetric NLS Equations: Let $r(t, x)=k \bar{q}(-t, x)$. This is an integrable equation

$$
a q_{t}(t, x)=-\frac{1}{2} q_{x x}(t, x)+k q^{2}(t, x) \bar{q}(-t, x),
$$

provided that $\bar{a}=a$. The recursion operator of this equation is

$$
\mathscr{R}=\left(\begin{array}{cc}
k q(t, x) D_{x}^{-1} \bar{q}(-t, x)-\frac{a}{2} D_{x} & q(t, x) D_{x}^{-1} q(t, x) \\
-k^{2} \bar{q}(-t, x) D_{x}^{-1} \bar{q}(-t, x) & -k \bar{q}(-t, x) D_{x}^{-1} q(t, x)+\frac{a}{2} D_{x}
\end{array}\right),
$$

and one-soliton solution is obtained by letting $k_{2}=\bar{k}_{1}$ where $k_{1}=\alpha+i \beta, \alpha, \beta \in \mathbb{R}$, and $e^{\delta_{2}}=k e^{\bar{\delta}_{1}}$ in $(13)$ as

$$
q(t, x)=\frac{e^{(\alpha+i \beta) x+\frac{(\alpha+i \beta)^{2}}{2 a} t+\delta_{1}}}{1-k \frac{e^{2 \alpha x+\frac{2 i \alpha \beta}{a} t+\delta_{1}+\bar{\delta}_{1}}}{4 \alpha^{2}}},
$$

for $\alpha \neq 0$ in Type 1 . To have a real-valued solution we consider $q(t, x) \bar{q}(t, x)=|q(t, x)|^{2}$. Here we have

$$
|q(t, x)|^{2}=\frac{16 \alpha^{4} e^{2 \alpha x+\frac{\alpha^{2}-\beta^{2}}{a} t+\delta_{1}+\bar{\delta}_{1}}}{\left(k e^{2 \alpha x+\delta_{1}+\bar{\delta}_{1}}-4 \alpha^{2} \cos \left(\frac{2 \alpha \beta}{a} t\right)\right)^{2}+16 \alpha^{4} \sin ^{2}\left(\frac{2 \alpha \beta}{a} t\right)} .
$$


When $\beta \neq 0$ and

$$
t=\frac{a n \pi}{2 \alpha \beta}, \quad k e^{2 \alpha x+\delta_{1}+\bar{\delta}_{1}}-4 \alpha^{2}(-1)^{n}=0,
$$

where $n$ is an integer, both focusing ( $\operatorname{sign}(k)=-1)$ and defocusing ( $\operatorname{sign}(k)=1)$ cases have singularities. When $\beta=0$ the focusing case is non-singular but asymptotically growing in time.

In Type 2, if we take $k_{1}=i \beta, k_{2}=i \gamma$ for $\beta, \gamma \in \mathbb{R}, e^{\delta_{1}}=a_{1}+i b_{1}$, and $e^{\delta_{2}}=a_{2}+i b_{2}$ for $a_{j}, b_{j} \in \mathbb{R}$, $j=1,2$ then one-soliton solution becomes

$$
q(t, x)=\frac{e^{i \beta x-\frac{\beta^{2}}{2 a} t}\left(a_{1}+i b_{1}\right)}{1+\frac{1}{(\beta+\gamma)^{2}} e^{i(\beta+\gamma) x+\frac{\left(\gamma^{2}-\beta^{2}\right)}{2 a} t}\left(a_{1}+i b_{1}\right)\left(a_{2}+i b_{2}\right)}, \quad \beta \neq-\gamma
$$

Hence the function $|q(t, x)|^{2}$ is

$$
|q(t, x)|^{2}=\frac{e^{\frac{\left(\gamma^{2}+\beta^{2}\right)}{2 a} t}\left(a_{1}^{2}+b_{1}^{2}\right)}{2\left[\cosh \left(\frac{\left(\gamma^{2}-\beta^{2}\right)}{2 a} t\right)+\cos \theta\right]},
$$

where

$$
\theta=(\beta+\gamma) x+\omega_{0}
$$

for $\omega_{0}=\arccos \left(\left(a_{1} a_{2}-b_{1} b_{2}\right) /(\beta+\gamma)^{2}\right)$ with $a_{1}^{2}+b_{1}^{2}=(\beta+\gamma)^{2} / k$ and $a_{2}^{2}+b_{2}^{2}=k(\beta+\gamma)^{2}$. Clearly, the solution is singular at $t=0$ and $\theta=(2 n+1) \pi, n$ integer and non-singular for $t \neq 0$.

2) S-Symmetric NLS Equations: Let $r(t, x)=k \bar{q}(t,-x)$. This is an integrable equation

$$
a q_{t}(t, x)=-\frac{1}{2} q_{x x}(t, x)+k q^{2}(t, x) \bar{q}(t,-x),
$$

provided that $\bar{a}=-a$. The recursion operator of this equation is

$$
\mathscr{R}=\left(\begin{array}{cc}
k q(t, x) D_{x}^{-1} \bar{q}(t,-x)-\frac{a}{2} D_{x} & q(t, x) D_{x}^{-1} q(t, x) \\
-k^{2} \bar{q}(t,-x) D_{x}^{-1} \bar{q}(t,-x) & -k \bar{q}(t,-x) D_{x}^{-1} q(t, x)+\frac{a}{2} D_{x}
\end{array}\right) .
$$

In Type 1 , one-soliton solution is obtained by letting $k_{2}=-\bar{k}_{1}$ where $k_{1}=\alpha+i \beta, \alpha, \beta \in \mathbb{R}, a=i y$, $y \in \mathbb{R}$, and $e^{\delta_{2}}=k e^{\bar{\delta}_{1}}$ in 13 as

$$
q(t, x)=\frac{e^{(\alpha+i \beta) x+\frac{(\alpha+i \beta)^{2}}{2 i y} t+\delta_{1}}}{1+k \frac{e^{2 i \beta x+\frac{2 \alpha \beta}{y} t+\delta_{1}+\bar{\delta}_{1}}}{4 \beta^{2}}}
$$

where $\beta \neq 0$. Hence the function $|q(t, x)|^{2}$ is

$$
|q(t, x)|^{2}=\frac{16 \beta^{4} e^{2 \alpha x+\frac{2 \alpha \beta}{y} t+\delta_{1}+\bar{\delta}_{1}}}{\left(k e^{\frac{2 \alpha \beta}{y} t+\delta_{1}+\bar{\delta}_{1}}+4 \beta^{2} \cos (2 \beta x)\right)^{2}+16 \beta^{4} \sin ^{2}(2 \beta x)} .
$$


If $\alpha \neq 0$ the above function is singular at

$$
x=\frac{n \pi}{2 \beta}, \quad k e^{\frac{2 \alpha \beta}{y} t+\delta_{1}+\bar{\delta}_{1}}+4 \beta^{2}(-1)^{n}=0,
$$

where $n$ is an integer, both for focusing and defocusing cases. If $\alpha=0$, the function (33) becomes

$$
|q(t, x)|^{2}=\frac{2 \beta^{2}}{k[B+\cos (2 \beta x)]},
$$

for $B=\left(\rho^{2}+16 \beta^{4}\right) /\left(8 \rho \beta^{2}\right)$ where $\rho=k e^{\delta_{1}+\bar{\delta}_{1}}$. Obviously, the solution 34 is non-singular if $B>1$ or $B<-1$.

Example 1. For the set of parameters $\left(k_{1}, k_{2}, e^{\delta_{1}}, e^{\delta_{2}}, k, a\right)=$ $(i, i, i,-i, 1, i / 2)$, we get the solution

$$
|q(t, x)|^{2}=\frac{16}{(17+8 \cos (2 x))} .
$$

This solution represents a periodic solution. Its graph is given in Figure 1.

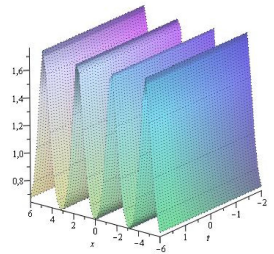

Figure 1: A periodic solution

For Type 2 if we let $a=i \alpha, \alpha \in \mathbb{R}, e^{\delta_{1}}=a_{1}+i b_{1}$, and $e^{\delta_{2}}=a_{2}+i b_{2}$ for $a_{j}, b_{j} \in \mathbb{R}, j=1,2$ then one-soliton solution becomes

$$
q(t, x)=\frac{e^{k_{1} x+i \frac{k_{1}^{2}}{2 \alpha} t}\left(a_{1}+i b_{1}\right)}{1-\frac{1}{\left(k_{1}+k_{2}\right)^{2}} e^{\left(k_{1}+k_{2}\right) x-i \frac{\left(k_{1}^{2}-k_{2}^{2}\right)}{2 \alpha} t}\left(a_{1}+i b_{1}\right)\left(a_{2}+i b_{2}\right)}, \quad k_{1} \neq-k_{2} .
$$

Therefore the function $|q(t, x)|^{2}$ is

$$
|q(t, x)|^{2}=\frac{e^{\left(k_{1}-k_{2}\right) x}\left(a_{1}^{2}+b_{1}^{2}\right)}{2\left[\cosh \left(\left(k_{1}+k_{2}\right) x\right)-\cos \theta\right]},
$$

where

$$
\theta=\frac{1}{2 \alpha}\left(k_{1}^{2}-k_{2}^{2}\right) t-\omega_{0}
$$

for $\omega_{0}=\arccos \left(\left(a_{1} a_{2}-b_{1} b_{2}\right) /\left(k_{1}+k_{2}\right)^{2}\right)$ with $a_{1}^{2}+b_{1}^{2}=-\left(k_{1}+k_{2}\right)^{2} / k$ and $a_{2}^{2}+b_{2}^{2}=-k\left(k_{1}+\right.$ $\left.k_{2}\right)^{2}$. The solution is singular at $x=0$ and $\theta=2 n \pi$ for $n$ integer, and non-singular for $x \neq 0$.

3) ST-Symmetric NLS Equations: Let $r(t, x)=k \bar{q}(-t,-x)$. This is an integrable equation

$$
a q_{t}(t, x)=-\frac{1}{2} q_{x x}(t, x)+k q^{2}(t, x) \bar{q}(-t,-x),
$$

provided that $\bar{a}=-a$. The recursion operator of this equation is

$$
\mathscr{R}=\left(\begin{array}{cc}
k q(t, x) D_{x}^{-1} \bar{q}(-t,-x)-\frac{a}{2} D_{x} & q(t, x) D_{x}^{-1} q(t, x) \\
-k^{2} \bar{q}(-t,-x) D_{x}^{-1} \bar{q}(-t,-x) & -k \bar{q}(-t,-x) D_{x}^{-1} q(t, x)+\frac{a}{2} D_{x}
\end{array}\right),
$$


and one-soliton solution is obtained by letting $k_{2}=-\bar{k}_{1}$ where $k_{1}=\alpha+i \beta, \alpha, \beta \in \mathbb{R}$ and $e^{\delta_{2}}=k e^{\bar{\delta}_{1}}$ in (13) as

$$
q(t, x)=\frac{e^{(\alpha+i \beta) x+\frac{(\alpha+i \beta)^{2}}{2 a} t+\delta_{1}}}{1+k \frac{e^{2 i \beta x+\frac{2 i \alpha \beta}{a} t+\delta_{1}+\bar{\delta}_{1}}}{4 \beta^{2}}}
$$

where $\beta \neq 0$ in Type 1 . Therefore $|q(t, x)|^{2}$ is

$$
|q(t, x)|^{2}=\frac{16 \beta^{4} e^{2 \alpha x+\frac{\left(\alpha^{2}-\beta^{2}\right)}{a} t+\delta_{1}+\bar{\delta}_{1}}}{\left(k e^{\delta_{1}+\bar{\delta}_{1}}+4 \beta^{2} \cos \left(2 \beta x+\frac{2 \alpha \beta}{a} t\right)\right)^{2}+16 \beta^{4} \sin ^{2}\left(2 \beta x+\frac{2 \alpha \beta}{a} t\right)} .
$$

This function is singular on the line $2 \beta x+(2 \alpha \beta t / a)=n \pi$ where $n$ is an integer, if the condition $k e^{\delta_{1}+\bar{\delta}_{1}}+4 \beta^{2}(-1)^{n}=0$ is satisfied by the parameters of the solution, otherwise it represents a non-singular wave solution for both focusing and defocusing cases. For $\alpha=0,(a>0)$, the solution represents a localized wave solution.

In Type 2, if we take $e^{\delta_{1}}=a_{1}+i b_{1}$ and $e^{\delta_{2}}=a_{2}+i b_{2}$ for $a_{j}, b_{j} \in \mathbb{R}, j=1,2$ we have the one-soliton solution as

$$
q(t, x)=\frac{e^{k_{1} x+\frac{k_{1}^{2}}{2 a} t}\left(a_{1}+i b_{1}\right)}{1-\frac{1}{\left(k_{1}+k_{2}\right)^{2}} e^{\left(k_{1}+k_{2}\right) x+\left(\frac{\left(k_{1}^{2}-k_{2}^{2}\right)}{2 a}\right) t}\left(a_{1}+i b_{1}\right)\left(a_{2}+i b_{2}\right)}, \quad k_{1} \neq-k_{2} .
$$

The corresponding function $|q(t, x)|^{2}$ is

$$
|q(t, x)|^{2}=\frac{e^{\phi}\left(a_{1}^{2}+b_{1}^{2}\right)}{1-2 \gamma e^{\theta}+e^{2 \theta}}
$$

where

$$
\phi=2 k_{1} x+\frac{k_{1}^{2}}{a} t, \quad \theta=\left(k_{1}+k_{2}\right) x+\frac{1}{2 a}\left(k_{1}^{2}-k_{2}^{2}\right) t,
$$

$\gamma=\left(a_{1} a_{2}-b_{1} b_{2}\right) /\left(k_{1}+k_{2}\right)^{2}, a_{1}^{2}+b_{1}^{2}=-\left(k_{1}+k_{2}\right)^{2} / k$, and $a_{2}^{2}+b_{2}^{2}=-k\left(k_{1}+k_{2}\right)^{2}$. The above function is singular when the function $f(\theta)=e^{2 \theta}-2 \gamma e^{\theta}+1$ vanishes. It becomes zero when $e^{\theta}=\gamma \pm \sqrt{\gamma^{2}-1}$. Hence if $\gamma<1$ the solution is non-singular.

\section{Standard and Nonlocal MKdV Equations}

Letting $a_{2}=0$ and $a_{3}=i / a$ we get the mKdV system

$$
\begin{aligned}
& a q_{t}=\frac{1}{4} q_{x x x}-\frac{3}{2} q r q_{x}, \\
& a r_{t}=\frac{1}{4} r_{x x x}-\frac{3}{2} q r r_{x} .
\end{aligned}
$$


This system has the same recursion operator (12) as the NLS system. One-soliton solution of the above system is [27]

$$
q(t, x)=\frac{e^{\theta_{1}}}{1+A e^{\theta_{1}+\theta_{2}}}, \quad r(t, x)=\frac{e^{\theta_{2}}}{1+A e^{\theta_{1}+\theta_{2}}},
$$

with $\theta_{i}=k_{i} x-\left(k_{i}^{3} t / 4 a\right)+\delta_{i}, i=1,2$, and $A=-1 /\left(k_{1}+k_{2}\right)^{2}$. Here $k_{1}, k_{2}, \delta_{1}$, and $\delta_{2}$ are arbitrary complex numbers. In $\mathrm{mKdV}$ case, there are also two types of approaches represented in [26] to find solutions of the standard $\mathrm{mKdV}$ and nonlocal $\mathrm{mKdV}$ (and cmKdV) equations.

1. MKdV Equations: Let $r(t, x)=k q(t, x)$ then $\mathrm{mKdV}$ system reduces to the integrable $\mathrm{mKdV}$ equation

$$
a q_{t}=\frac{1}{4} q_{x x x}-\frac{3 k}{2} q^{2} q_{x}
$$

In Type 1 one-soliton solution is obtained by letting $k_{1}=k_{2}=\alpha+i \beta$ and $e^{\delta_{2}}=k e^{\delta_{1}}=a_{1}+i b_{1}$ for $\alpha, \beta, a_{1}, b_{1} \in \mathbb{R}$ in 45 as

$$
q(t, x)=\frac{e^{(\alpha+i \beta) x-\frac{\left(\alpha^{3}-3 \alpha \beta^{2}\right)+i\left(3 \alpha^{2} \beta-\beta^{3}\right)}{4 a} t}\left(a_{1}+i b_{1}\right)}{1-\frac{k}{4\left(\alpha^{2}+\beta^{2}\right)^{2}} e^{2(\alpha+i \beta) x-\frac{\left(\alpha^{3}-3 \alpha \beta^{2}\right)+i\left(3 \alpha^{2} \beta-\beta^{3}\right)}{2 a} t}\left(a_{1}+i b_{1}\right)^{2}(\alpha-i \beta)^{2}} .
$$

Therefore we obtain the function

$$
|q(t, x)|^{2}=\frac{Y}{W}
$$

where

$$
\begin{aligned}
Y & =e^{2 \alpha x-\frac{\left(\alpha^{3}-3 \alpha \beta^{2}\right)}{2 a} t}\left(a_{1}^{2}+b_{1}^{2}\right) \\
W & =1-\gamma_{1} \cos \theta+\frac{\gamma_{1}^{2}}{4} e^{\phi}=\frac{\gamma_{1}^{2}}{4}\left[\frac{4}{\gamma_{1}^{2}}\left(1-\gamma_{1} \cos \theta\right)+e^{\phi}\right]
\end{aligned}
$$

where

for

$$
\theta=2 \beta x-\frac{1}{2 a}\left(3 \alpha^{2} \beta-\beta^{3}\right) t+\omega_{0}, \quad \phi=4 \alpha x-\frac{1}{a}\left(\alpha^{3}-3 \alpha \beta^{2}\right) t
$$

$$
\omega_{0}=\arccos \left(\left(\left(a_{1} \alpha+b_{1} \beta\right)^{2}-\left(a_{1} \beta-b_{1} \alpha\right)^{2}\right) /\left(a_{1}^{2}+b_{1}^{2}\right)\left(\alpha^{2}+\beta^{2}\right)\right)
$$

and $\gamma_{1}=k\left(a_{1}^{2}+b_{1}^{2}\right) / 2\left(\alpha^{2}+\beta^{2}\right)$. Hence we conclude that if $\left|\gamma_{1}\right| \leq 1$ the solution (48) is nonsingular. Type 2 approach gives $k_{1}=k_{2}=0$ yielding trivial solution.

2. CmKdV Equations: Let $r=k \bar{q}(t, x)$ then $\mathrm{mKdV}$ system reduces to the integrable cmKdV equation

$$
a q_{t}=\frac{1}{4} q_{x x x}-\frac{3 k}{2} q \bar{q} q_{x}
$$

where $\bar{a}=a$. One-soliton solution is obtained by letting $k_{2}=\bar{k}_{1}=\alpha-i \beta$ for $\alpha, \beta \in \mathbb{R}$ and $e^{\delta_{2}}=$ $k e^{\bar{\delta}_{1}}$ in 45 in Type 1 as

$$
q(t, x)=\frac{e^{(\alpha+i \beta) x-\frac{\left(\alpha^{3}-3 \alpha \beta^{2}\right)+i\left(3 \alpha^{2} \beta-\beta^{3}\right)}{4 a} t+\delta_{1}}}{1-\frac{k}{4 \alpha^{2}} e^{2 \alpha x+\frac{\left(3 \alpha \beta^{2}-\alpha^{3}\right)}{2 a} t+\delta_{1}+\bar{\delta}_{1}}},
$$


so the function $|q(t, x)|^{2}$ is

$$
|q(t, x)|^{2}=\frac{e^{2 \alpha x-\frac{\left(\alpha^{3}-3 \alpha \beta^{2}\right)}{2 a} t+\delta_{1}+\bar{\delta}_{1}}}{\left(1-\frac{k}{4 \alpha^{2}} e^{\left.2 \alpha x+\frac{\left(3 \alpha \beta^{2}-\alpha^{3}\right)}{2 a} t+\delta_{1}+\bar{\delta}_{1}\right)^{2}} .\right.}
$$

For $k<0$, the solution (52) can be written as

$$
|q(t, x)|^{2}=-\frac{\alpha^{2}}{k} \operatorname{sech}^{2}\left(\alpha x+\frac{\left(3 \alpha \beta^{2}-\alpha^{3}\right)}{4 a} t+\frac{\delta_{1}+\bar{\delta}_{1}}{2}+\delta\right),
$$

where $\delta=\ln \left(-k / 4 \alpha^{2}\right) / 2$. The above solution is non-singular.

We obtain a different one-soliton solution in Type 2 under the constraints $k_{1}=-\bar{k}_{1}, k_{2}=-\bar{k}_{2}$, $A k e^{\delta_{1}+\bar{\delta}_{1}}=1$, and $A e^{\delta_{2}+\bar{\delta}_{2}}=k$ used in 45 . If we let $k_{1}=\alpha i, k_{2}=\beta i, e^{\delta_{1}}=a_{1}+i b_{1}$, and $e^{\delta_{2}}=$ $a_{2}+i b_{2}$ for $\alpha, \beta, a_{j}, b_{j} \in \mathbb{R}, j=1,2$, one-soliton solution becomes

$$
q(t, x)=\frac{e^{i \alpha x+i \frac{\alpha^{3}}{4 a} t}\left(a_{1}+i b_{1}\right)}{1+\frac{1}{(\alpha+\beta)^{2}} e^{i(\alpha+\beta) x+i \frac{\left(\alpha^{3}+\beta^{3}\right)}{4 a} t}\left(a_{1}+i b_{1}\right)\left(a_{2}+i b_{2}\right)},
$$

hence the corresponding function $|q(t, x)|^{2}$ is

$$
|q(t, x)|^{2}=\frac{a_{1}^{2}+b_{1}^{2}}{4} \sec ^{2}\left(\frac{\theta}{2}\right)
$$

where

$$
\theta=(\alpha+\beta) x+\frac{1}{4 a}\left(\alpha^{3}+\beta^{3}\right) t+\omega_{0}
$$

for $\omega_{0}=\arccos \left(\left(a_{1} a_{2}-b_{1} b_{2}\right) /(\alpha+\beta)^{2}\right)$ with $a_{1}^{2}+b_{1}^{2}=(\alpha+\beta)^{2} / k$ and $a_{2}^{2}+b_{2}^{2}=k(\alpha+\beta)^{2}$. This is a singular solution for $\theta=(2 n+1) \pi, n$ is an integer.

There are also two different types of nonlocal reductions.

1. Nonlocal MKdV Equations: Let $r=k q\left(\mu_{1} t, \mu_{2} x\right)$ then $\mathrm{mKdV}$ system reduces to the integrable nonlocal $\mathrm{mKdV}$ equation

$$
a q_{t}(t, x)=\frac{1}{4} q_{x x x}(t, x)-\frac{3 k}{2} q(t, x) q\left(\mu_{1} t, \mu_{2} x\right) q_{x}(t, x),
$$

provided that $\mu_{1} \mu_{2}=1$. There is only one possibility $\left(\mu_{1}, \mu_{2}\right)=(-1,-1)$. If we consider the Type 1 approach, we get $k_{1}=-k_{2}$ which gives trivial solution $q(t, x)=0$. In Type 2, one-soliton solution is obtained from 45 with the parameters satisfying the relations $A k e^{2 \delta_{1}}=1$ and $A e^{2 \delta_{2}}=k$ as

$$
q(t, x)=\frac{i \sigma_{1} e^{k_{1} x-\frac{k_{1}^{3}}{4 a} t}\left(k_{1}+k_{2}\right)}{\sqrt{k}\left(1+\sigma_{1} \sigma_{2} e^{\left(k_{1}+k_{2}\right) x-\frac{\left(k_{1}^{3}+k_{2}^{3}\right)}{4 a} t}\right)}, \quad \sigma_{j}= \pm 1, \quad j=1,2 .
$$


If we let $a \in \mathbb{R}, k_{1}=\alpha_{1}+i \beta_{1}$, and $k_{2}=\alpha_{2}+i \beta_{2}$ then we obtain the solution $|q(t, x)|^{2}$ corresponding to 57 ) as

$$
|q(t, x)|^{2}=\frac{e^{\theta}}{2 k\left[\cosh (\phi)+\sigma_{1} \sigma_{2} \cos (\varphi)\right]},
$$

where $\theta=\left(\alpha_{1}-\alpha_{2}\right) x-\left(\left(\alpha_{1}^{3}-3 \alpha_{1} \beta_{1}^{2}-\alpha_{2}^{3}+3 \alpha_{2} \beta_{2}^{2}\right) t / 4 a\right), \phi=A_{1} x+B_{1} t$, and $\varphi=A_{2} x+B_{2} t$. Here

$$
\begin{aligned}
& A_{1}=\alpha_{1}+\alpha_{2}, \quad B_{1}=-\frac{1}{4 a}\left(\alpha_{1}^{3}-3 \alpha_{1} \beta_{1}^{2}+\alpha_{2}^{3}-3 \alpha_{2} \beta_{2}^{2}\right), \\
& A_{2}=\beta_{1}+\beta_{2}, \quad B_{2}=\frac{1}{4 a}\left(\beta_{1}^{3}-3 \alpha_{1}^{2} \beta_{1}+\beta_{2}^{3}-3 \alpha_{2}^{2} \beta_{2}\right) .
\end{aligned}
$$

There are cases where the solution (58) is nonsingular:

(a) If we have $k_{1}=k_{2}$ for real $k_{1}$ and $\sigma_{1} \sigma_{2}=1$ then the solution (57) becomes

$$
q(t, x)=\frac{i \sigma_{1} k_{1}}{\sqrt{k}} \operatorname{sech}\left(k_{1} x-\frac{k_{1}^{3}}{4 a} t\right) .
$$

(b) If $B_{1} A_{2}=B_{2} A_{1}$ then the solution $(58)$ becomes

$$
|q(t, x)|^{2}=\frac{e^{\theta}}{2 k\left[\cosh (\phi)+\sigma_{1} \sigma_{2} \cos \left(\frac{B_{2}}{B_{1}} \phi\right)\right]} .
$$

Example 2. If we take $\left(k_{1}, k_{2}, \sigma_{1}, \sigma_{2} k, a\right)=(i, 1+(i / 2), 1,1,-1,1 / 4)$ then we have the solution

$$
|q(t, x)|^{2}=\frac{13 e^{-u}}{8[\cosh (u)+\cos (3 u / 2)]}
$$

where $u=x-t / 4$. This is a complexiton solution. The graph of this solution is given in Figure 2.

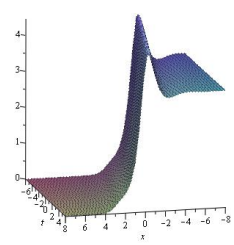

Figure 2: A complexiton solution

2. Nonlocal CmKdV Equations: Let $r=k \bar{q}\left(\mu_{1} t, \mu_{2} x\right)$ then $\mathrm{mKdV}$ system reduces to the integrable nonlocal $\mathrm{cmKdV}$ equation

$$
a q_{t}(t, x)=\frac{1}{4} q_{x x x}(t, x)-\frac{3 k}{2} q(t, x) \bar{q}\left(\mu_{1} t, \mu_{2} x\right) q_{x}(t, x),
$$

provided that $\bar{a}=\mu_{1} \mu_{2} a$. One-soliton solution is obtained by letting $k_{2}=\mu_{2} \bar{k}_{1}$ and $e^{\delta_{2}}=k e^{\bar{\delta}_{1}}$ in Type 1. In Type 2, a different one-soliton solution is obtained by letting $k_{1}=-\bar{k}_{1} \mu_{2}, k_{2}=-\bar{k}_{2} \mu_{2}$, $A k e^{\delta_{1}+\bar{\delta}_{1}}=1$, and $A e^{\delta_{2}+\bar{\delta}_{2}}=k$. In this case there are three possibilities $\left(\mu_{1}, \mu_{2}\right)=\{(-1,1),(1,-1),(-1,-1)\}$. Hence we have three integrable nonlocal $\mathrm{cmKdV}$ equations: 
2.i) T-Symmetric Nonlocal CmKdV Equations: Let $r=k \bar{q}(-t, x)$ then $\mathrm{mKdV}$ system reduces to the nonlocal $\mathrm{cmKdV}$ equation

$$
a q_{t}(t, x)=-\frac{1}{4} q_{x x x}(t, x)+\frac{3}{2} k \bar{q}(-t, x) q(t, x) q_{x}(t, x), \quad \bar{a}=-a .
$$

In Type 1 if we let $a=i b$, for nonzero $b \in \mathbb{R}, k_{1}=\alpha+i \beta$ so $k_{2}=\alpha-i \beta$ for $\alpha, \beta \in \mathbb{R}, \alpha \neq 0$ then one-soliton solution becomes

$$
q(t, x)=\frac{e^{(\alpha+i \beta) x+\frac{i\left(\alpha^{3}-3 \alpha \beta^{2}\right)-3 \alpha^{2} \beta+\beta^{3}}{4 b} t+\delta_{1}}}{1-\frac{k}{4 \alpha^{2}} e^{2 \alpha x+i \frac{\alpha^{3}-3 \alpha \beta^{2}}{2 b} t+\delta_{1}+\bar{\delta}_{1}}} .
$$

The corresponding function $|q(t, x)|^{2}$ is

$$
|q(t, x)|^{2}=\frac{e^{2 \alpha x+\frac{\left(\beta^{3}-3 \alpha^{2} \beta\right)}{2 b} t+\delta_{1}+\bar{\delta}_{1}}}{\left[\frac{k}{4 \alpha^{2}} e^{2 \alpha x+\delta_{1}+\bar{\delta}_{1}}-\cos \left(\frac{\left(\alpha^{3}-3 \alpha \beta^{2}\right)}{2 b} t\right)\right]^{2}+\sin ^{2}\left(\frac{\left(\alpha^{3}-3 \alpha \beta^{2}\right)}{2 b} t\right)}
$$

When $\alpha^{3}-3 \alpha \beta^{2} \neq 0$ and

$$
t=\frac{2 n b \pi}{\left(\alpha^{3}-3 \alpha \beta^{2}\right)}, \quad \frac{k}{4 \alpha^{2}} e^{2 \alpha x+\delta_{1}+\bar{\delta}_{1}}-(-1)^{n}=0,
$$

where $n$ is an integer, for both focusing and defocusing cases, the solution is singular. When $\alpha^{3}-3 \alpha \beta^{2}=0$ the solution for focusing case is non-singular. When $\alpha=0$ the solution is exponentially growing for $\beta^{3} / b>0$ and exponentially decaying for $\beta^{3} / b<0$.

In Type 2 if we let $a=i \alpha, k_{1}=i \beta, k_{2}=i \gamma$ for $\alpha, \beta, \gamma \in \mathbb{R}$, and $e^{\delta_{1}}=a_{1}+i b_{1}, e^{\delta_{2}}=a_{2}+i b_{2}$ for $a_{j}, b_{j} \in \mathbb{R}, j=1,2$ then one-soliton solution becomes

$$
q(t, x)=\frac{e^{i \beta x+\frac{\beta^{3}}{4 \alpha} t}\left(a_{1}+i b_{1}\right)}{1+\frac{1}{(\beta+\gamma)^{2}} e^{i(\beta+\gamma) x+\frac{\left(\beta^{3}+\gamma^{3}\right)}{4 \alpha} t}\left(a_{1}+i b_{1}\right)\left(a_{2}+i b_{2}\right)} .
$$

Hence the function $|q(t, x)|^{2}$ is

$$
|q(t, x)|^{2}=\frac{e^{\frac{\left(\beta^{3}-\gamma^{3}\right)}{4 \alpha} t}\left(a_{1}^{2}+b_{1}^{2}\right)}{2\left[\cosh \left(\frac{\left(\beta^{3}+\gamma^{3}\right)}{4 \alpha} t\right)+\cos \theta\right]},
$$

where

$$
\theta=(\beta+\gamma) x+\omega_{0}
$$

for $\omega_{0}=\arccos \left(\left(a_{1} a_{2}-b_{1} b_{2}\right) /(\beta+\gamma)^{2}\right)$ with $a_{1}^{2}+b_{1}^{2}=(\beta+\gamma)^{2} / k, a_{2}^{2}+b_{2}^{2}=k(\beta+\gamma)^{2}$, and $\beta \neq-\gamma$. This solution is singular only at $t=0, \theta=(2 n+1) \pi$ for $n$ integer. 
2.ii) S-Symmetric Nonlocal CmKdV Equations: Let $r=k \bar{q}(t,-x)$ then $\mathrm{mKdV}$ system reduces to the nonlocal $\mathrm{cmKdV}$ equation

$$
a q_{t}(t, x)=-\frac{1}{4} q_{x x x}(t, x)+\frac{3}{2} k \bar{q}(t,-x) q(t, x) q_{x}(t, x), \quad \bar{a}=-a .
$$

If we consider Type 1 and let $a=i b$ for nonzero $b \in \mathbb{R}, k_{1}=\alpha+i \beta$ and so $k_{2}=-\alpha+i \beta$ for $\alpha, \beta \in \mathbb{R}, \beta \neq 0$ then one-soliton solution becomes

$$
q(t, x)=\frac{e^{(\alpha+i \beta) x+\frac{i \alpha^{3}-3 \alpha^{2} \beta-3 i \alpha \beta^{2}+\beta^{3}}{4 b} t+\delta_{1}}}{1+\frac{k}{4 \beta^{2}} e^{2 i \beta x+i \frac{\alpha^{3}-3 \alpha \beta^{2}}{2 b} t+\delta_{1}+\bar{\delta}_{1}}}
$$

and so $|q(t, x)|^{2}$ is

$$
|q(t, x)|^{2}=\frac{e^{2 \alpha x+\frac{\left(\beta^{3}-3 \alpha^{2} \beta\right)}{2 b} t+\delta_{1}+\bar{\delta}_{1}}}{\left[\frac{k}{4 \beta^{2}} e^{\frac{\left(\beta^{3}-3 \alpha^{2} \beta\right)}{2 b} t+\delta_{1}+\bar{\delta}_{1}}+\cos (2 \beta x)\right]^{2}+\sin ^{2}(2 \beta x)} .
$$

For $x=n \pi /(2 \beta)$ and $k e^{\left(\beta^{3}-3 \alpha^{2} \beta\right) t / 2 b+\delta_{1}+\bar{\delta}_{1}} /\left(4 \beta^{2}\right)+(-1)^{n}=0$, where $n$ is an integer, the solution is unbounded but for $\beta^{2}=3 \alpha^{2}$ and $k e^{\delta_{1}+\bar{\delta}_{1}} /\left(4 \beta^{2}\right)+(-1)^{n} \neq 0$ we have a periodical solution. For $\alpha=0$, the solution $(69)$ becomes

$$
|q(t, x)|^{2}=\frac{e^{\delta_{1}+\bar{\delta}_{1}}}{\gamma\left[\sigma_{k} \cosh \left(\frac{\beta^{3}}{2 b} t+\ln \left(\frac{|\gamma|}{2}\right)\right)+\cos (2 \beta x)\right]},
$$

where $\gamma=k e^{\delta_{1}+\bar{\delta}_{1}} /\left(2 \beta^{2}\right), \sigma_{k}=1$ if $k>0$, and $\sigma_{k}=-1$ if $k<0$. This solution is non-singular for $|\gamma|>2, \beta^{3} / b>0$ and $|\gamma|<2, \beta^{3} / b<0$ for any $t \geq 0$.

For Type 2 if we let $a=i \alpha, \alpha \in \mathbb{R}, e^{\delta_{1}}=a_{1}+i b_{1}$, and $e^{\delta_{2}}=a_{2}+i b_{2}$ for $a_{j}, b_{j} \in \mathbb{R}, j=1,2$ we obtain the one-soliton solution as

$$
q(t, x)=\frac{e^{k_{1} x+i \frac{k_{1}^{3}}{4 \alpha} t}\left(a_{1}+i b_{1}\right)}{1-\frac{1}{\left(k_{1}+k_{2}\right)^{2}} e^{\left(k_{1}+k_{2}\right) x+i \frac{\left(k_{1}^{3}+k_{2}^{3}\right)}{4 \alpha} t}\left(a_{1}+i b_{1}\right)\left(a_{2}+i b_{2}\right)} .
$$

Therefore the function $|q(t, x)|^{2}$ is

$$
|q(t, x)|^{2}=\frac{e^{\left(k_{1}-k_{2}\right) x}\left(a_{1}^{2}+b_{1}^{2}\right)}{2\left[\cosh \left(\left(k_{1}+k_{2}\right) x\right)+\cos \theta\right]},
$$

where

$$
\theta=\frac{1}{4}\left(k_{1}^{3}+k_{2}^{3}\right) t-\omega_{0}
$$

for $\omega_{0}=\arccos \left(\left(b_{1} b_{2}-a_{1} a_{2}\right) /\left(k_{1}+k_{2}\right)^{2}\right)$ with $a_{1}^{2}+b_{1}^{2}=-\frac{\left(k_{1}+k_{2}\right)^{2}}{k}$ and $a_{2}^{2}+b_{2}^{2}=-k\left(k_{1}+k_{2}\right)^{2}$, $k_{1} \neq-k_{2}$. This solution has singularity at $x=0, \theta=(2 n+1) \pi$ for $n$ integer. 
2.iii) ST-Symmetric Nonlocal CmKdV Equations: Let $r=k \bar{q}(-t,-x)$ then $\mathrm{mKdV}$ system reduces to the nonlocal $\mathrm{cmKdV}$ equation

$$
a q_{t}(t, x)=-\frac{1}{4} q_{x x x}(t, x)+\frac{3}{2} k \bar{q}(-t,-x) q(t, x) q_{x}(t, x), \quad \bar{a}=a .
$$

In Type 1 if we let $k_{1}=\alpha+i \beta$ and so $k_{2}=-\alpha+i \beta$ for $\alpha, \beta \in \mathbb{R}, \beta \neq 0$ the one-soliton solution $q(t, x)$ becomes

$$
q(t, x)=\frac{e^{(\alpha+i \beta) x-\frac{\alpha^{3}+3 \alpha^{2} i \beta-3 \alpha \beta^{2}-i \beta^{3}}{4 a} t+\delta_{1}}}{1+\frac{k}{4 \beta^{2}} e^{2 i \beta x-i \frac{\left(6 \alpha^{2} \beta-2 \beta^{3}\right)}{4 a} t+\delta_{1}+\bar{\delta}_{1}}} .
$$

Then we obtain the function $|q(t, x)|^{2}$ as

$$
|q(t, x)|^{2}=\frac{e^{\theta}}{\mu\left[\left(\frac{1}{\mu}+\frac{\mu}{4}\right)+\cos \phi\right]},
$$

where

$$
\theta=2 \alpha x+\frac{1}{2 a}\left(3 \alpha \beta^{2}-\alpha^{3}\right) t+\delta_{1}+\bar{\delta}_{1}, \phi=2 \beta x+\frac{1}{2 a}\left(\beta^{3}-3 \alpha^{2} \beta\right) t,
$$

and $\mu=k e^{\delta_{1}+\bar{\delta}_{1}} /\left(2 \beta^{2}\right)$. This solution is non-singular for all $\mu$ except $\mu= \pm 2$.

For Type 2 , if we take $e^{\delta_{1}}=a_{1}+i b_{1}$ and $e^{\delta_{2}}=a_{2}+i b_{2}$ for $a_{j}, b_{j} \in \mathbb{R}, j=1,2$ we obtain the one-soliton solution as

$$
q(t, x)=\frac{e^{k_{1} x-\frac{k_{1}^{3}}{4 a} t}\left(a_{1}+i b_{1}\right)}{1-\frac{1}{\left(k_{1}+k_{2}\right)^{2}} e^{\left(k_{1}+k_{2}\right) x-\frac{\left(k_{1}^{3}+k_{2}^{3}\right)}{4 a} t}\left(a_{1}+i b_{1}\right)\left(a_{2}+i b_{2}\right)},
$$

hence the function $|q(t, x)|^{2}$ is

$$
|q(t, x)|^{2}=\frac{e^{\phi}}{1-2 \gamma e^{\theta}+e^{2 \theta}}
$$

where

$$
\theta=\left(k_{1}+k_{2}\right) x-\frac{1}{4 a}\left(k_{1}^{3}+k_{2}^{3}\right) t, \phi=2 k_{1} x-\frac{k_{1}^{3}}{2 a} t,
$$

$\gamma=\left(a_{1} a_{2}-b_{1} b_{2}\right) /\left(k_{1}+k_{2}\right)^{2}$ with $a_{1}^{2}+b_{1}^{2}=-\left(k_{1}+k_{2}\right)^{2} / k$, and $a_{2}^{2}+b_{2}^{2}=-k\left(k_{1}+k_{2}\right)^{2}, k_{1} \neq-k_{2}$. The above function has singularity when $e^{\theta}=\gamma \pm \sqrt{\gamma^{2}-1}$. Hence for $\gamma<1$ and $k_{2}>k_{1}$ the solution is non-singular and bounded.

Example 3. For the set of the parameters $\left(k_{1}, k_{2}, e^{\delta_{1}}, e^{\delta_{2}}\right.$, $k, a)=\left(\frac{1}{2}, \frac{1}{4},-\frac{3}{4}, \frac{3}{4},-1,2\right)$ we obtain the following asymptotically decaying soliton

$$
q(t, x)=\frac{\left(-3 e^{\frac{1}{2} x-\frac{1}{64} t}\right)}{4\left(1+e^{\frac{3}{4} x-\frac{9}{512} t}\right)}
$$

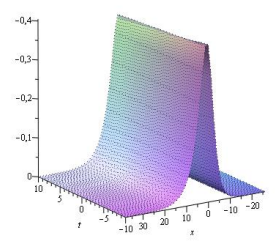

Figure 3: An asymptotically decaying soliton 
whose graph is given in Figure 3.

Remark. All dynamical variables considered so far are complex valued functions. We claim that all the results presented here will be valid if the dynamical variables are pseudo complex valued functions. Any pseudo complex number is $\alpha=a+i b$ where $i^{2}=1$. Complex conjugation is $\bar{\alpha}=$ $a-i b$. Hence the norm of a pseudo complex number is not positive definite $\alpha \bar{\alpha}=a^{2}-b^{2}$. NLS equation

$$
i q_{t}=-\frac{1}{2} q_{x x}+k q^{2} \bar{q}
$$

has real and imaginary parts $(q=u+i v)$

$$
\begin{aligned}
u_{t} & =-\frac{1}{2} v_{x x}+k\left(u^{2}-\varepsilon v^{2}\right) v \\
\varepsilon v_{t} & =-\frac{1}{2} u_{x x}+k\left(u^{2}-\varepsilon v^{2}\right) u,
\end{aligned}
$$

where $i^{2}=\varepsilon= \pm 1$

\section{Fordy-Kulish System}

Systems of integrable nonlinear partial differential equations arise when the Lax pairs are given in certain Lie algebras. Fordy-Kulish (FK) system of equations are examples of such equations [28], [29]. We briefly give the Lax representations of these equations,

$$
\begin{aligned}
\phi_{x} & =\left(\lambda H_{S}+Q^{A} E_{A}\right) \phi \\
\phi_{t} & =\left(A^{a} H_{a}+B^{A} E_{A}+C^{D} E_{D}\right) \phi
\end{aligned}
$$

where the dynamical variables are $Q_{A}=\left(q^{\alpha}, p^{\alpha}\right)$, the functions $A^{a}, B^{A}$, and $C^{A}$ depend on the spectral parameter $\lambda$, on the dynamical variables $\left(q^{\alpha}, p^{\alpha}\right)$, and their $x$-derivatives. The system of FK equations is an example when the functions $A, B$, and $C$ are quadratic functions of $\lambda$. Let $q^{\alpha}(t, x)$ and $p^{\alpha}(t, x)$ be the complex dynamical variables where $\alpha=1,2, \cdots, N$, then the FK integrable system arising from the integrability condition of Lax equations (79) and (80) is given by

$$
\begin{aligned}
& a q_{t}^{\alpha}=q_{x x}^{\alpha}+R_{\beta \gamma-\delta}^{\alpha} q^{\beta} q^{\gamma} p^{\delta}, \\
& a p_{t}^{\alpha}=p_{x x}^{\alpha}+R_{-\beta-\gamma \delta}^{-\alpha} p^{\beta} p^{\gamma} q^{\delta},
\end{aligned}
$$

for all $\alpha=1,2, \cdots, N$. Here $R_{\beta \gamma-\delta}^{\alpha}$ and $R_{-\beta-\gamma \delta}^{-\alpha}$ are the curvature tensors of a Hermitian symmetric space satisfying

$$
\left(R_{\beta \gamma-\delta}^{\alpha}\right)^{\star}=R_{-\beta-\gamma \delta}^{-\alpha},
$$

and $a$ is a complex number. These equations are known as the FK system which is integrable in the sense that they are obtained from the zero curvature condition of a connection defined on a 
Hermitian symmetric space and these equations can also be written in a Hamiltonian form.

The standard reduction of the above FK system is obtained by letting $p^{\alpha}=k\left(q^{\alpha}\right)^{\star}$ for all $\alpha=$ $1,2, \cdots, N$. The FK system (81) reduces to a single equation

$$
a q_{t}^{\alpha}=q_{x x}^{\alpha}+k R_{\beta \gamma-\delta}^{\alpha} q^{\beta} q^{\gamma}\left(q^{\delta}\right)^{\star}, \quad \alpha=1,2, \cdots, N,
$$

provided that $a^{\star}=-a$ and $(83)$ is satisfied. Here $*$ over a letter denotes complex conjugation.

\section{Nonlocal Fordy-Kulish Equations}

Here we will show that the Fordy-Kulish system is compatible with the nonlocal reduction of Ablowitz-Musslimani type. For this purpose using a similar constraint as in NLS system we let

$$
p^{\alpha}(t, x)=k\left[q^{\alpha}\left(\mu_{1} t, \mu_{2} x\right)\right]^{\star}, \quad \alpha=1,2, \cdots, N,
$$

where $\mu_{1}^{2}=\mu_{2}^{2}=1$. Under this constraint the FK system 81 - 82 reduces to the following system of equations:

$$
a q_{t}^{\alpha}(t, x)=q_{x x}^{\alpha}(t, x)+k R_{\beta \gamma-\delta}^{\alpha} q^{\beta}(t, x) q^{\gamma}(t, x)\left(q^{\delta}\left(\mu_{1} t, \mu_{2} x\right)\right)^{\star}
$$

provided that $a^{\star}=-\mu_{1} a$ and $(83)$ is satisfied. In addition to 86 we have also an equation for $q^{\delta}\left(\mu_{1} t, \mu_{2} x\right)$ which can be obtained by letting $t \rightarrow \mu_{1} t, x \rightarrow \mu_{2} x$ in 86. Hence we obtain Tsymmetric, S-symmetric, and ST-symmetric nonlocal FK equations. Nonlocal reductions correspond to $\left(\mu_{1}, \mu_{2}\right)=\{(-1,1),(1,-1),(-1,-1)\}$. Hence corresponding to these values of $\mu_{1}$ and $\mu_{2}$ we have three different nonlocal integrable FK equations. They are given as follows:

1. T-Symmetric Nonlocal FK Equations:

$$
a q_{t}^{\alpha}(t, x)=q_{x x}^{\alpha}(t, x)+k R_{\beta \gamma-\delta}^{\alpha} q^{\beta}(t, x) q^{\gamma}(t, x)\left(q^{\delta}(-t, x)\right)^{\star},
$$

with $a^{\star}=a$.

2. S-Symmetric Nonlocal FK Equations:

$$
a q_{t}^{\alpha}(t, x)=q_{x x}^{\alpha}(t, x)+k R_{\beta \gamma-\delta}^{\alpha} q^{\beta}(t, x) q^{\gamma}(t, x)\left(q^{\delta}(t,-x)\right)^{\star},
$$

with $a^{\star}=-a$.

3. ST-Symmetric Nonlocal FK Equations:

$$
a q_{t}^{\alpha}(t, x)=q_{x x}^{\alpha}(t, x)+k R_{\beta \gamma-\delta}^{\alpha} q^{\beta}(t, x) q^{\gamma}(t, x)\left(q^{\delta}(-t,-x)\right)^{\star},
$$

with $a^{\star}=a$. All these three nonlocal equations are integrable. 


\section{Super Integrable Systems}

When the Lax pair, in $(1+1)$-dimensions, is given in a super Lie algebra the resulting evolution equations are super integrable systems. They are given as a coupled system

$$
\begin{aligned}
& q_{t}^{i}=F^{i}\left(q^{k}, \varepsilon^{k}, q_{x}^{k}, \varepsilon_{x}^{k}, q_{x x}^{k}, \varepsilon_{x x}^{k}, \cdots\right), \\
& \varepsilon_{t}^{i}=G^{i}\left(q^{k}, \varepsilon^{k}, q_{x}^{k}, \varepsilon_{x}^{k}, q_{x x}^{k}, \varepsilon_{x x}^{k}, \cdots\right),
\end{aligned}
$$

for all $i=1,2, \cdots, N$ where $F^{i}$ and $G^{i}(i=1,2, \cdots, N)$ are functions of the dynamical variables $q^{i}(t, x), \varepsilon^{i}(t, x)$, and their partial derivatives with respect to $x$. Here $q^{i}$, s are bosonic and $\varepsilon^{i}$, s are the fermionic dynamical variables. Since we start with a super Lax pair then the system (90)-(91) is a super integrable system of nonlinear partial differential equations.

\section{Nonlocal Super NLS and MKdV Equations}

As an example taking the Lax pair in super $s l(2, R)$ algebra we obtain the super AKNS system. We have two bosonic $(q, r)$ and two fermionic $(\varepsilon, \beta)$ dynamical variables. They satisfy the following evolution equations [30]-[32],

Bosonic Equations:

$$
\begin{aligned}
q_{t}= & a_{2}\left(-\frac{1}{2} q_{x x}+q^{2} r+2 \varepsilon_{x} \varepsilon+2 q \beta \varepsilon\right)+i a_{3}\left(-\frac{1}{4} q_{x x x}+\frac{3}{2} q r q_{x}+3\left(\varepsilon_{x} \varepsilon\right)_{x}\right. \\
& \left.-3 q \beta_{x} \varepsilon+3 q \beta \varepsilon_{x}\right), \\
r_{t}= & a_{2}\left(\frac{1}{2} r_{x x}-q r^{2}+2 \beta_{x} \beta-2 r \beta \varepsilon\right)+i a_{3}\left(-\frac{1}{4} r_{x x x}+\frac{3}{2} q r r_{x}-3\left(\beta_{x} \beta\right)_{x}\right. \\
& \left.+3 r \beta_{x} \varepsilon-3 r \beta \varepsilon_{x}\right),
\end{aligned}
$$

Fermionic Equations:

$$
\begin{aligned}
\beta_{t}= & a_{2}\left(\beta_{x x}-r \varepsilon_{x}-\frac{1}{2} \varepsilon r_{x}-\frac{1}{2} q r \beta\right)+i a_{3}\left(-\beta_{x x x}+\frac{3}{4} r q_{x} \beta+\frac{3}{4} q r_{x} \beta+\frac{3}{2} q r \beta_{x}\right. \\
& \left.+\frac{3}{2} r_{x} \varepsilon_{x}+\frac{3}{4} \varepsilon r_{x x}\right), \\
\varepsilon_{t}= & a_{2}\left(-\varepsilon_{x x}+q \beta_{x}+\frac{1}{2} \beta q_{x}+\frac{1}{2} q r \varepsilon\right)+i a_{3}\left(-\varepsilon_{x x x}+\frac{3}{4} r q_{x} \varepsilon+\frac{3}{4} q r_{x} \varepsilon+\frac{3}{2} q r \varepsilon_{x}\right. \\
& \left.+\frac{3}{2} q_{x} \beta_{x}+\frac{3}{4} \beta q_{x x}\right),
\end{aligned}
$$

where $a_{2}$ and $a_{3}$ are arbitrary constants. 


\subsection{Super NLS Equations}

Letting $a_{3}=0$ in the equations (92)-(95) we get the super coupled NLS system of equations. There are two bosonic $(q, r)$ and two fermionic $(\varepsilon, \beta)$ potentials satisfying

$$
\begin{aligned}
a q_{t} & =-\frac{1}{2} q_{x x}+q^{2} r+2 \varepsilon_{x} \varepsilon+2 q \beta \varepsilon, \\
a r_{t} & =\frac{1}{2} r_{x x}-q r^{2}+2 \beta_{x} \beta-2 r \beta \varepsilon, \\
a \varepsilon_{t} & =-\varepsilon_{x x}+q \beta_{x}+\frac{1}{2} \beta q_{x}+\frac{1}{2} q r \varepsilon, \\
a \beta_{t} & =\beta_{x x}-r \varepsilon_{x}-\frac{1}{2} \varepsilon r_{x}-\frac{1}{2} q r \beta,
\end{aligned}
$$

where $a_{2}=1 / a$. The standard reduction is $r=k_{1} \bar{q}$ and $\beta=k_{2} \bar{\varepsilon}$ where $k_{1}$ and $k_{2}$ are constants, a bar over a quantity denotes the Berezin conjugation in the Grassmann algebra. If $P$ and $Q$ are super functions (bosonic or fermionic) then $\overline{P Q}=\bar{Q} \bar{P}$. Under these constraints the above equations 96- -99 reduce to the following super NLS equations provided $k_{1}=k_{2}^{2}$ and $\bar{a}=-a$,

$$
\begin{aligned}
& a q_{t}=-\frac{1}{2} q_{x x}+k_{1} q^{2} \bar{q}+2 \varepsilon_{x} \varepsilon+2 k_{2} q \bar{\varepsilon} \varepsilon, \\
& a \varepsilon_{t}=-\varepsilon_{x x}+k_{2} q \bar{\varepsilon}_{x}+\frac{1}{2} k_{2} \bar{\varepsilon} q_{x}+\frac{1}{2} k_{1} q \bar{q} \varepsilon .
\end{aligned}
$$

Here we show that super NLS system (96)-99] can be reduced to nonlocal super NLS equations. This can be done by choosing the super Ablowitz-Musslimani reduction as

$$
r(t, x)=k_{1} \bar{q}\left(\mu_{1} t, \mu_{2} x\right), \quad \beta(t, x)=k_{2} \bar{\varepsilon}\left(\mu_{1} t, \mu_{2} x\right) .
$$

where $\mu_{1}^{2}=\mu_{2}^{2}=1$. Here $k_{1}$ and $k_{2}$ are real constants. Under these constraints the above set 96)(99) reduces to super NLS equations [33], [34],

$$
\begin{aligned}
a q_{t}(t, x)= & -\frac{1}{2} q_{x x}(t, x)+k_{1} q^{2}(t, x) \bar{q}\left(\mu_{1} t, \mu_{2} x\right)+2 \varepsilon_{x}(t, x) \varepsilon(t, x) \\
& +2 k_{2} q(t, x) \bar{\varepsilon}\left(\mu_{1} t, \mu_{2} x\right) \varepsilon(t, x), \\
a \varepsilon_{t}(t, x)= & -\varepsilon_{x x}(t, x)+k_{2} q(t, x) \bar{\varepsilon}_{x}\left(\mu_{1} t, \mu_{2} x\right)+\frac{1}{2} k_{2} \bar{\varepsilon}\left(\mu_{1} t, \mu_{2} x\right) q_{x}(t, x) \\
& +\frac{1}{2} k_{1} q(t, x) \bar{q}\left(\mu_{1} t, \mu_{2} x\right) \varepsilon(t, x),
\end{aligned}
$$

provided that

$$
\bar{a} \mu_{1}=-a, \quad k_{2}^{2} \mu_{2}=k_{1} .
$$

Nonlocal reductions correspond to the choices $\left(\mu_{1}, \mu_{2}\right)=\{(-1,1),(1,-1),(-1,-1)\}$. They are explicitly given by,

1. T-Symmetric Nonlocal Super NLS Equations:

$$
a q_{t}(t, x)=-\frac{1}{2} q_{x x}(t, x)+k_{1} q^{2}(t, x) \bar{q}(-t, x)+2 \varepsilon_{x}(t, x) \varepsilon(t, x)
$$




$$
\begin{aligned}
& +2 k_{2} q(t, x) \bar{\varepsilon}(-t, x), \varepsilon(t, x), \\
a \varepsilon_{t}(t, x)= & -\varepsilon_{x x}(t, x)+k_{2} q(t, x) \bar{\varepsilon}_{x}(-t, x)+\frac{1}{2} k_{2} \bar{\varepsilon}(-t, x) q_{x}(t, x) \\
& +\frac{1}{2} k_{1} q(t, x) \bar{q}(-t, x) \varepsilon(t, x),
\end{aligned}
$$

with $a^{\star}=a$ and $k_{1}=k_{2}^{2}$.

2. S-Symmetric Nonlocal Super NLS Equations:

$$
\begin{aligned}
a q_{t}(t, x)= & -\frac{1}{2} q_{x x}(t, x)+k_{1} q^{2}(t, x) \bar{q}(t,-x)+2 \varepsilon_{x}(t, x) \varepsilon(t, x)+2 k_{2} q(t, x) \bar{\varepsilon}(t,-x) \varepsilon(t, x), \\
a \varepsilon_{t}(t, x)= & -\varepsilon_{x x}(t, x)+k_{2} q(t, x) \bar{\varepsilon}_{x}(t,-x)+\frac{1}{2} k_{2} \bar{\varepsilon}(t,-x) q_{x}(t, x) \\
& +\frac{1}{2} k_{1} q(t, x) \bar{q}(t,-x) \varepsilon(t, x),
\end{aligned}
$$

with $a^{\star}=-a$ and $k_{1}=-k_{2}^{2}$.

3. ST-Symmetric Nonlocal Super NLS Equations:

$$
\begin{aligned}
a q_{t}(t, x)= & -\frac{1}{2} q_{x x}(t, x)+k_{1} q^{2}(t, x) \bar{q}(-t,-x)+2 \varepsilon_{x}(t, x) \varepsilon(t, x) \\
& +2 k_{2} q(t, x) \bar{\varepsilon}(-t,-x), \varepsilon(t, x), \\
a \varepsilon_{t}(t, x)= & -\varepsilon_{x x}(t, x)+k_{2} q(t, x) \bar{\varepsilon}_{x}(-t,-x)+\frac{1}{2} k_{2} \bar{\varepsilon}(-t,-x) q_{x}(t, x) \\
& +\frac{1}{2} k_{1} q(t, x) \bar{q}(-t,-x) \varepsilon(t, x),
\end{aligned}
$$

with $a^{\star}=a$ and $k_{1}=-k_{2}^{2}$.

\subsection{Super MKdV Systems}

Another special case of the super AKNS equations is the super mKdV system [30], [31]

$$
\begin{aligned}
a q_{t} & =-\frac{1}{4} q_{x x x}+\frac{3}{2} r q q_{x}+3\left(\varepsilon_{x} \varepsilon\right)_{x}-3 q \beta_{x} \varepsilon+3 q \beta \varepsilon_{x}, \\
a r_{t} & =-\frac{1}{4} r_{x x x}+\frac{3}{2} r q r_{x}-3\left(\beta_{x} \beta\right)_{x}+3 r \beta_{x} \varepsilon-3 r \beta \varepsilon_{x}, \\
a \varepsilon_{t} & =-\varepsilon_{x x x}+\frac{3}{4}(r q)_{x} \varepsilon+\frac{3}{2} q r \varepsilon_{x}+\frac{3}{2} q_{x} \beta_{x}+\frac{3}{4} \beta q_{x x}, \\
a \beta_{t} & =-\beta_{x x x}+\frac{3}{4}(r q)_{x} \beta+\frac{3}{2} q r \beta_{x}+\frac{3}{2} r_{x} \varepsilon_{x}+\frac{3}{4} \varepsilon r_{x x} .
\end{aligned}
$$

The standard reduction is $r=k_{1} \bar{q}, \beta=k_{2} \bar{\varepsilon}$. Then we obtain [30],

$$
\begin{aligned}
a q_{t} & =-\frac{1}{4} q_{x x x}+\frac{3}{2} k_{1} \bar{q} q q_{x}+3\left(\varepsilon_{x} \varepsilon\right)_{x}-3 k_{2} q \bar{\varepsilon}_{x} \varepsilon+3 k_{2} q \bar{\varepsilon} \varepsilon_{x}, \\
a \varepsilon_{t} & =-\varepsilon_{x x x}+\frac{3}{4} k_{1}(\bar{q} q)_{x} \varepsilon+\frac{3}{2} k_{1} q \bar{q} \varepsilon_{x}+\frac{3}{2} k_{2} q_{x} \bar{\varepsilon}_{x}+\frac{3}{4} k_{2} \bar{\varepsilon} q_{x x},
\end{aligned}
$$


provided that $k_{1}=k_{2}^{2}$ and $\bar{a}=a$. For the super mKdV system, Ablowitz-Musslimani type of reduction is also possible. Letting

$$
r(t, x)=k_{1} \bar{q}\left(\mu_{1} t, \mu_{2} x\right), \quad \beta(t, x)=k_{2} \bar{\varepsilon}\left(\mu_{1} t, \mu_{2} x\right),
$$

where $\mu_{1}^{2}=\mu_{2}^{2}=1$ we get the following system of equations

$$
\begin{aligned}
a q_{t}(t, x)= & -\frac{1}{4} q_{x x x}(t, x)+\frac{3}{2} k_{1} \bar{q}\left(\mu_{1} t, \mu_{2} x\right) q(t, x) q_{x}(t, x)+3\left(\varepsilon_{x}(t, x) \varepsilon(t, x)\right)_{x} \\
& -3 q(t, x) \bar{\varepsilon}_{x}\left(\mu_{1} t, \mu_{2} x\right) \varepsilon(t, x)+3 k_{2} q(t, x) \bar{\varepsilon}\left(\mu_{1} t, \mu_{2} x\right) \varepsilon_{x}(t, x), \\
a \varepsilon_{t}(t, x)= & -\varepsilon_{x x x}(t, x)+\frac{3}{4} k_{1}\left(\bar{q}\left(\mu_{1} t, \mu_{2} x\right) q(t, x)\right)_{x} \varepsilon(t, x)+\frac{3}{2} k_{1} q(t, x) \bar{q}\left(\mu_{1} t, \mu_{2} x\right) \varepsilon_{x}(t, x) \\
& +\frac{3}{2} k_{2} q_{x}(t, x) \bar{\varepsilon}_{x}\left(\mu_{1} t, \mu_{2} x\right)+\frac{3}{4} k_{2} \bar{\varepsilon}\left(\mu_{1} t, \mu_{2} x\right) q_{x x}(t, x),
\end{aligned}
$$

provided that $\bar{a} \mu_{1} \mu_{2}=a, k_{2}^{2} \mu_{2}=k_{1}$. Nonlocal reductions correspond to the choices $\left(\mu_{1}, \mu_{2}\right)=$ $\{(-1,1),(1,-1),(-1,-1)\}$. They are explicitly given by,

1. T-Symmetric Nonlocal Super MKdV Equations: Here $\bar{a}=-a$ and $k_{1}=k_{2}^{2}$.

$$
\begin{aligned}
a q_{t}(t, x)= & -\frac{1}{4} q_{x x x}(t, x)+\frac{3}{2} k_{1} \bar{q}(-t, x) q(t, x) q_{x}(t, x)+3\left(\varepsilon_{x}(t, x) \varepsilon(t, x)\right)_{x} \\
& -3 q(t, x) \bar{\varepsilon}_{x}(-t, x) \varepsilon(t, x)+3 k_{2} q(t, x) \bar{\varepsilon}(-t, x) \varepsilon_{x}(t, x), \\
a \varepsilon_{t}(t, x)= & -\varepsilon_{x x x}(t, x)+\frac{3}{4} k_{1}(\bar{q}(-t, x) q(t, x))_{x} \varepsilon(t, x)+\frac{3}{2} k_{1} q(t, x) \bar{q}(-t, x) \varepsilon_{x}(t, x) \\
& +\frac{3}{2} k_{2} q_{x}(t, x) \bar{\varepsilon}_{x}(-t, x)+\frac{3}{4} k_{2} \bar{\varepsilon}(-t, x) q_{x x}(t, x),
\end{aligned}
$$

2. S-Symmetric Nonlocal Super MKdV Equations: Here $\bar{a}=-a$ and $k_{1}=-k_{2}^{2}$.

$$
\begin{aligned}
a q_{t}(t, x)= & -\frac{1}{4} q_{x x x}(t, x)+\frac{3}{2} k_{1} \bar{q}(t,-x) q(t, x) q_{x}(t, x)+3\left(\varepsilon_{x}(t, x) \varepsilon(t, x)\right)_{x} \\
& -3 q(t, x) \bar{\varepsilon}_{x}(t,-x) \varepsilon(t, x)+3 k_{2} q(t, x) \bar{\varepsilon}(t,-x) \varepsilon_{x}(t, x), \\
a \varepsilon_{t}(t, x)= & -\varepsilon_{x x x}(t, x)+\frac{3}{4} k_{1}(\bar{q}(t,-x) q(t, x))_{x} \varepsilon(t, x)+\frac{3}{2} k_{1} q(t, x) \bar{q}(t,-x) \varepsilon_{x}(t, x) \\
& +\frac{3}{2} k_{2} q_{x}(t, x) \bar{\varepsilon}_{x}(t,-x)+\frac{3}{4} k_{2} \bar{\varepsilon}(t,-x) q_{x x}(t, x),
\end{aligned}
$$

3. ST-Symmetric Nonlocal Super MKdV Equations: Here $\bar{a}=a$ and $k_{1}=-k_{2}^{2}$.

$$
\begin{aligned}
a q_{t}(t, x)= & -\frac{1}{4} q_{x x x}(t, x)+\frac{3}{2} k_{1} \bar{q}(-t,-x) q(t, x) q_{x}(t, x)+3\left(\varepsilon_{x}(t, x) \varepsilon(t, x)\right)_{x} \\
& -3 q(t, x) \bar{\varepsilon}_{x}(-t,-x) \varepsilon(t, x)+3 k_{2} q(t, x) \bar{\varepsilon}(-t,-x) \varepsilon_{x}(t, x), \\
a \varepsilon_{t}(t, x)= & -\varepsilon_{x x x}(t, x)+\frac{3}{4} k_{1}(\bar{q}(-t,-x) q(t, x))_{x} \varepsilon(t, x)+\frac{3}{2} k_{1} q(t, x) \bar{q}(-t,-x) \varepsilon_{x}(t, x) \\
& +\frac{3}{2} k_{2} q_{x}(t, x) \bar{\varepsilon}_{x}(-t,-x)+\frac{3}{4} k_{2} \bar{\varepsilon}(-t,-x) q_{x x}(t, x),
\end{aligned}
$$




\section{Concluding Remarks}

In this work we first presented all integrable nonlocal reductions of NLS and MKdV systems. We gave the recursion operators and the soliton solutions of these nonlocal equations. We then presented the extension of the nonlocal NLS equation to nonlocal Fordy-Kulish equations on symmetric spaces. Starting with the super AKNS system we studied all possible nonlocal reductions and found two new super integrable systems. They are the nonlocal super NLS equations and nonlocal super $\mathrm{mKdV}$ systems of equations. There are three different nonlocal types of super integrable equations. They correspond to T-, S-, and ST- symmetric super NLS and super mKdV equations.

From the study of NLS and mKdV systems (both bosonic and fermionic integrable systems) we observed that they have standard and nonlocal reductions. Moreover in both of these systems there are at least one nonlocal reduction to a standard reduction. For instance both systems have $r(t, x)=k \bar{q}(t, x)$ as a standard reduction and the corresponding nonlocal reductions are $r(t, x)=k \bar{q}\left(\mu_{1} t, \mu_{2} x\right)$ where $k$ is real constant and $\left(\mu_{1}, \mu_{2}\right)=(1,-1),(-1,1),(-1,-1)$. From these reductions we obtain standard and nonlocal NLS equations and standard and nonlocal complex $\mathrm{mKdV}$ equations and their nonlocal super integrable extensions. The $\mathrm{mKdV}$ system has additional standard and nonlocal reductions. Standard reduction $r(t, x)=k q(t, x), k$ is real constant, and its corresponding nonlocal reduction $r(t, x)=k q(-t,-x)$ give the nonlocal $\mathrm{mKdV}$ equation. From all these experiences we conclude with a conjecture: If a system of equations admits a standard reduction then there exists at least one corresponding nonlocal reduction of the same system.

\section{Acknowledgment}

This work is partially supported by the Scientific and Technological Research Council of Turkey (TÜBİTAK).

\section{References}

[1] Ablowitz, M.J., Musslimani, Z.H.: Integrable nonlocal nonlinear Schrödinger equation. Phys. Rev. Lett. 110, 064105 (2013)

[2] Ablowitz, M.J., Musslimani, Z.H.: Inverse scattering transform for the integrable nonlocal nonlinear Schrödinger equation. Nonlinearity 29, 915-946 (2016)

[3] Ablowitz, M.J., Musslimani, Z.H.: Integrable nonlocal nonlinear equations. Studies in Applied Mathematics 139, Issue: 1, 7-59 (2016)

[4] Fokas, A.S.: Integrable multidimensional versions of the nonlocal Schrödinger equation. Nonlinearity 29, 319-324 (2016) 
[5] Sakkaravarthi, K., Kanna, T.: Bright solitons in coherently coupled nonlinear Schrödinger equations with alternate signs of nonlinearities. J. Math. Phys. 54, 013701 (2013)

[6] Gerdjikov, V.S., Saxena, A.: Complete integrability of nonlocal nonlinear Schrödinger equation. J. Math. Phys. 58, Issue:1, 013502 (2017) (arXiv:1510.00480[nlin.SI])

[7] Sinha, D., Ghosh, P.K.: Integrable nonlocal vector nonlinear Schrödinger equation with selfinduced parity-time symmetric potential. Phys. Lett. A381, 124-128 (2017)

[8] Gerdjikov, V.S., Grahovski, D.G., Ivanov, R.I.: On the integrable wave interactions and Lax pairs on symmetric spaces. (arXiv:1607.06940[nlin.SI]), in the special issue on Mathematical modelling and physical dynamics of solitary waves: From continuum mechanics to field theory, Eds. Ivan C. Christov, M.D. Todorov, S. Yoshida. (Wave Motion, http://dx.doi.org/10.1016/j.wavemoti.2016.07.012)

[9] Gerdjikov, V.S., Grahovski, D.G., Ivanov, R.I.: On the N-wave equations with PT symmetry. Theor. and Math. Phys. 188, No.3, 1305-1321 (2016)

[10] Gerdjikov, V.S.: On nonlocal models of Kulish-Sklyanin type and generalized Fourier transforms. Stud. Comp. Int. 681, 37-52 (2017) (arXiv:1703.03705[nlin.SI])

[11] Khare, A., Saxena, A.: Periodic and hyperbolic soliton solutions of a number of nonlocal nonlinear equations. J. Math. Phys. 56, 032104 (2015)

[12] Li, M., Xu, T.: Dark and antidark soliton interactions in the nonlocal nonlinear Schrödinger equation with the self-induced parity-time-symmetric potential. Phys. Rev. E 91, 033202 (2015)

[13] Huang, X., King, L.: Soliton solutions for the nonlocal nonlinear Schrödinger equation. Eur. Phys. J. Plus 131, 148 (2016)

[14] Wen, X.Y., Yan, Z., Yang, Y.: Dynamics of higher-order rational solitons for the nonlocal nonlinear Schrödinger equation with the self-induced parity-time-symmetric potential. Chaos 26, 063123 (2015)

[15] Caudrelier, V.: Interplay between the inverse scattering method and the unified transform with an application. (arXiv:1704.05306[math-ph])

[16] Yang, J.: General N-solitons and their dynamics in several nonlocal nonlinear Schrödinger equations. (arXiv:1712.01181 [nlin.SI])

[17] Feng, B.F., Luo, X.D., Ablowitz, M.J., Musslimani, Z.H.: General soliton solution to a nonlocal nonlinear Schrödinger equation with zero and nonzero boundary conditions. (arXiv:1712.09172 [nlin.SI]) 
[18] Ma, L.Y., Shen, S.F., Zhu, Z.N.: Integrable nonlocal complex mKdV equation: soliton solution and gauge equivalence. (arXiv:1612.06723 [nlin.SI])

[19] Ji, J.L., Zhu, Z.N.: On a nonlocal modified Korteweg-de Vries equation: Integrability, Darboux transformation and soliton solutions. Commun. Non. Sci. Numer. Simulat. 42, 699-708 (2017)

[20] Ji, J.L., Zhu, Z.N.: Soliton solutions of an integrable nonlocal modified Korteweg-de Vries equation through inverse scattering transform. J. Math. An. and App. 453, 973-984 (2017) (arXiv:1603.03994 [nlin.SI])

[21] Yang, B., Yang, J.: Transformations between nonlocal and local integrable equations. (arXiv:1705.00332v1 [nlin.PS])

[22] Chen, K., Deng X., Lou S., Zhang D.: Solutions of nonlocal equations reduced from the AKNS hierarchy, to appear in Stud. App. Math., (2018) (arXiv:1710.10479 [nlin.SI])

[23] Gürses, M.: Nonlocal Fordy-Kulish equations on symmetric spaces. Phys. Lett. A 381, 17911794 (2017)

[24] Gürses, M., Pekcan, A.: Nonlocal nonlinear Schrödinger equations and their soliton solutions. (arXiv:1707.07610 [nlin.SI])

[25] Ablowitz, M.J., Kaup, D.J., Newell, A.C., Segur H.: The inverse scattering transform-Fourier analysis for nonlinear problems, Stud. Appl. Math. 53, Issue: 4, 249-315 (1974)

[26] Gürses, M., Pekcan, A.: Nonlocal nonlinear modified KdV equations and their soliton solutions. (arXiv:1711.01588 [nlin.SI])

[27] Iwao, M., Hirota, R.: Soliton solutions of a coupled modified KdV equations. J. Phys. Soc. Japan 66, No: 3, 577-588 (1997)

[28] Fordy, A.P, Kulish P.P.: Nonlinear Schrödinger equations and simple Lie algebras, Commun. Math. Phys. 89, 427-443 (1983)

[29] Fordy, A.P.: Derivative nonlinear Schrödinger equations and hermitian symmetric spaces, J. Phys. A: Math. Gen. 17, 1235-1245 (1984)

[30] Gürses, M., Og̃uz, Ö.: A super AKNS scheme. Phys. Lett. A 108, Issue: 9, 437-440 (1985)

[31] Gürses, M., Og̃uz, Ö.: A super soliton connection. Lett. in Math. Phys. 11, 235-246 (1986)

[32] Gürses, M., Og̃uz, Ö., Salihog̃lu, S.: Nonlinear partial differential equations on homogeneous spaces. Int. J. Mod. Phys. A 5, 1801-1817 (1990) 
[33] Kupperschmidt, B.A.: A super Korteweg-de Vries equation: An integrable system. Phys. Lett. 102A, 213 (1983)

[34] Kupperschmidt, B.A.: Bosons and fermions interacting integrably with the Korteweg-de Vries field. J. Phys. A: Math. Gen. 17, L869, (1984) 